\title{
Simón Rodríguez y Bolívar: dos lecturas político educativas para América
}

\author{
MAXIMILIANO DURÁN* \\ Universidad de Buenos Aires, Buenos Aires, Argentina
}

\section{RESUMEN}

El presente trabajo se propone discutir una interpretación tradicional sobre las figuras de Bolívar y Rodríguez relativas a la educación del pueblo. De acuerdo con la mayoría de los trabajos académicos escritos existiría una identificación política e intelectual entre Bolívar y Rodríguez. Dicha comunión se expresaría en las ideas que cada uno de ellos sostiene respecto de la educación del pueblo. Este artículo es crítico de esta interpretación tradicional y plantea que las ideas relativas a la educación son bien diferentes en ambos pensadores. A lo largo del trabajo se presentan críticamente los distintos trabajos que plantean esta comunidad de ideas, se analizan las similitudes y diferencias en ambos autores a la luz de los documentos conservados y se propone un momento conclusivo en el que se retoman los ejes centrales del texto.

\section{PALABRAS CLAVE}

historia de la educación; América Latina; Simón Rodríguez; Bolívar; Lancaster.

* Bolsista de pós-doutorado da Fundação de Amparo à Pesquisa do Estado do Rio de Janeiro (FAPERJ). 


\title{
SIMON RODRÍGUEZ AND BOLIVAR: TWO POSSIBLE POLITICAL AND EDUCATIONAL READINGS FOR AMERICA
}

\begin{abstract}
This paper discusses a traditional interpretation of the figures of Bolívar and Rodríguez on popular education. According to most academic papers written, there would be a political and intellectual identification between Bolivar and Rodríguez. This communion is expressed in the ideas that each holds for the education of the people. This article is critical of this traditional interpretation and argues that the ideas concerning education are very different for both thinkers. Throughout this work, the different works presented by this community of ideas are critically presented, the similarities and differences of both authors in the light of the documents kept are analyzed and a concluding moment in which the central axes of the text are resumed is proposed.
\end{abstract}

KEYWORDS

history of education; Latin America; Simón Rodríguez; Bolívar; Lancaster.

\section{SIMON RODRÍGUEZ E BOLIVAR: DUAS LEITURAS POLITICO-EDUCATIVAS PARA AMÉRICA}

RESUMO

Este trabalho se propõe a discutir uma interpretação tradicional da educação do povo nas obras de Rodríguez e Bolívar. Segundo a maioria dos trabalhos acadêmicos escritos, existiria uma identificação política e intelectual entre Bolívar e Rodríguez. Essa identificação se expressaria nas ideias que cada um deles sustenta sobre a educação do povo. $\mathrm{O}$ artigo que aqui apresentamos é crítico dessa interpretação tradicional, afirmando que as ideias ligadas à educação são bem diferentes em ambos os pensadores. Ao longo deste trabalho, apresentamos criticamente os diferentes trabalhos apresentados por essa comunidade de ideias, analizamos as semelhanças e as diferenças nos dois autores com base nos documentos conservados, e finalmente, propomos um momento conclusivo em que sejam retomados os eixos centrais do texto.

história da educação; América Latina; Simon Rodríguez; Bolívar; Lancaster. 


\section{INTRODUCCIÓN}

Los trabajos tradicionales dedicados al estudio de la obra de Rodríguez ${ }^{1}$ y Bolívar $^{2}$ coinciden de manera casi unánime en afirmar la existencia de un vínculo indisoluble en materia educativa entre ambos pensadores. ${ }^{3}$ Para la gran mayoría de los autores tanto Bolívar como Rodríguez piensan la educación del pueblo de manera casi idéntica. Esta afirmación transmitida de manera incuestionada en todos los trabajos consultados se apoya en dos puntos. El primero de ellos es el

1 Simón Rodríguez (1769-1854). Maestro y filósofo latinoamericano nacido en la ciudad de Caracas. Luego de un período cercano a treinta años en Europa decide volver a América para trabajar a favor de la causa emancipatoria americana. Se conoce muy poco sobre su estancia europea. Por algunas referencias epistolares, sabemos que recorrió a pie Francia e Italia en la compañía de Bolívar. Llegados a la Roma, Bolívar decide retornar a América para luchar contra el poder español. Rodríguez permanece en Europa, allí recorre Alemania, Francia, Italia, Rusia e Inglaterra. Un dato interesante es que en todos los países que vive trabaja como maestro de escuela. Retorna al continente americano en 1823. Es convocado por Bolívar en 1824 para unirse al ejército libertador. Con las tropas del ejército emprende desde Perú un viaje hasta la actual Bolivia. Allí es nombrado ministro de Educación y se le encomienda la creación de un plan de educación para la naciente república. El proyecto de educación popular creado pro Rodríguez es uno de los proyectos educativos más radicales y originales que se hayan escrito en el continente americano. A partir del despliegue de un principio igualitario sin precedentes recibe en un mismo salón de clases a todos los niños y niñas de la ciudad en calidad de iguales, sin distinción alguna. De acuerdo al maestro los niños no iban a la escuela para tornarse iguales sino al contrario, iban a la escuela porque eran iguales. Dicho proyecto es atacado por las oligarquías criollas desde diversos frentes. Rodríguez recibe insultos y calumnias. Luego de seis meses de ataques constantes presenta su renuncia ante el presidente de la república a su cargo. De Bolivia emprende un viaje que lo llevará a Chile, Ecuador, Colombia y Perú. En todos estos viajes Rodríguez trabaja como maestro,intenta publicar sus escritos con distinta suerte y proclama la necesidad de una educación republicana para todos y todas. En 1854, cansado, olvidado y en la más angustiante pobreza muere en el pueblo peruano de Amotape. Sus restos descansan hoy en día en el Panteón Nacional de Venezuela, a los pies de su amigo Simón Bolívar.

2 Simón Bolívar (1783-1830). Político y pensador venezolano. Uno de los próceres máximos del continente americano. Lideró y emprendió, desde inicios del siglo XIX las guerras de emancipación del poder español. Para ello empeñó todos sus bienes y fortuna. Padre de la Independencia de Colombia, Venezuela, Ecuador, Perú y Bolivia. En 1823 organizó y se hizo cargo del Ejército Unido Libertador que logró las victorias de Junín y Ayacucho (1824) que pusieron fin al poderío español en América. Escribió proyectos constitucionales para Bolivia, Colombia y Venezuela. Bolívar soñaba con un continente unido y soberano por medio de grandes federaciones. Las diversas oligarquías nacionalistas se opusieron abiertamente a sus planes. Fue perseguido, calumniado e insultado por aquellos que en tiempos de guerra fueron sus aliados y subalternos. En 1830 abandona la vida política y se retira a la isla de Santa Marta donde muere el 17 de diciembre de 1830.

3 Por cuestiones de espacio resulta imposible referencias todos los trabajos que sostienen esta postura. Entre los más destacados es posible mencionar los trabajos clásicos de Amunátegui (1876), Lozano y Lozano (1913), Mancini (1914), André (1924), Cova (1947), Grases (1953), Achury Valenzuela (1954), Álvarez Freites (1966), Rumazo González (1976), Briceño Porras (1991), Molins (1998), Jáuregui Olazábal (2000) y Lasheras (2004). 
magisterio que Rodríguez lleva a cabo en Europa cuando Bolívar es un joven viudo entregado a los placeres de la vida mundana entre 1804-1806. De acuerdo con todas las biografías de Rodríguez el maestro rescata a un Bolívar perdido y abatido por la pérdida de su esposa. Juntos emprenden un viaje a pie por Europa que finaliza en el Juramento del Monte Sacro. ${ }^{4}$ De acuerdo con el trabajo de la mayoría de los historiadores durante estos dos años Rodríguez habría culminado la formación de Bolívar. Para la tradición académica, el viaje de Rodríguez y Bolívar por el viejo continente es interpretado como un proceso iniciático en el que el viejo maestro ilustrado y republicano, pasa a su discípulo las ideas que más tarde materializa en el campo de batalla y la gestión política en América.

El segundo punto sobre el que la tradición se apoya para sostener la identidad de Rodríguez y Bolívar en materia educativa es el intercambio de ideas entre ambos a partir de 1823. Sobre la base de la carta de Pativilca se esgrimen una serie de ideas relativas a las concepciones educativas de ambos personajes. Castellanos (2007, p. 143) sostiene, que ambos personajes conciben un proyecto educativo singular para América en su viaje desde Perú hasta Bolivia. Para este autor, el viaje de Lima a Chuquisaca significa un proceso creativo en el que ambos personajes intercambian ideas y llevan adelante proyectos específicos vinculados con la educación. Para apoyar estas ideas recurre a una serie de presupuestos y a las palabras de O'Leary (1879-1888). Entre los distintos presupuestos que sostiene Castellanos (2007, p. 153) se encuentra la participación directa e indirecta de Rodríguez en la redacción de decretos relativos a la educación y en el empeño del maestro por convencer al Libertador de la importancia de asignar mayores recursos a las escuelas. A su vez, a partir de los escritos de Alfonso Rumazo González (1976), Lee Lofstrom (1987) y de O’Leary sostiene que Bolívar consulta todo con el maestro antes de tomar una decisión. Motivo por el cual Rodríguez se habría ganado la enemistad y encono de muchos personajes influyentes. De hecho, sostiene que el conflicto entre el Mariscal de Ayacucho y el maestro se inicia durante este período y por estas causas.

Según Castellanos, durante esta época en la que Rodríguez se erige como el responsable autorizado en materia educativa dentro del séquito de Bolívar, Sucre habría presentado a Bolívar un plan integral de educación popular para Cochabamba. Dicho proyecto habría sido presentado a Rodríguez quien lo habría analizado y realizado una serie de sugerencias que, según él supone, habrían molestado a Sucre (Castellanos, 2007, p. 166). ${ }^{5}$ Más allá de la veracidad de la anécdota y de las posibles causas del enojo de Sucre con Rodríguez interesa resaltar la interpretación que Castellanos hace de la participación de Rodríguez en la escena política. Según su punto de vista, Rodríguez goza de un altísimo prestigio intelectual y afectivo por parte del Libertador. Debido a ello es consultado en todos los temas relativos a la

4 Todas las biografías sobre Rodríguez coinciden en este punto. Para este tema en particular, ver Lozano y Lozano (1913), Álvarez Freites (1966), Rumazo González (1976), Jáuregui Olazábal (2000), Lasheras (2004) Castellanos (2007) y Vera Peñaranda (2009).

5 Hasta dónde hemos podido averiguar, no hay documentos que acrediten las afirmaciones de Castellanos sobre este supuesto enojo de Sucre. 
educación del pueblo. Él tiene, según Castellanos, una autoridad omnímoda en materia educativa. Él es el encargado de pensar y presentar a las autoridades un proyecto de educación popular. Autores como Âlvarez Freites (1966), Rumazo González (1976), Rojas (2008), Briggs (2010), entre muchos otros coinciden con esta descripción. Para ellos el estrecho vínculo entre el maestro y el libertador genera una serie de consecuencias en el ámbito de la gestión y participación política.

Ronald Briggs (2010, p. 5) sostiene al respecto que Rodríguez es uno de los responsables de la concientización política de Bolívar. Al igual que la mayoría de la tradición académica otorga al maestro un lugar importante en el éxito de Bolívar (Briggs, 2010, p. 6). De acuerdo con su punto de vista, en su rol de maestro, Rodríguez forma consciente y explícitamente a Bolívar para transformarlo en el Libertador. Debido a ello hay una conexión singular entre ambos en los temas relativos a la educación y la política a seguir. El caso más extremo en este tema es el que plantea Rojas (2008). Para este autor, la conexión es tan grande que llega a plantear una suerte de simbiosis. Según su interpretación, no habría diferencias entre el pensamiento de Bolívar y Rodríguez.

Es importante destacar que, ninguno de los textos consultados se apoya sobre una base documental amplia, ni precisa. Todos ellos se refieren a un conjunto de cartas y decretos escritos entre 1823 y 1826. En ellos lo único que se puede observar es el afecto entre ambos autores y el rol administrativo que ocupa Rodríguez en la naciente república de Bolivia. La documentación referida es insuficiente para sostener la coincidencia entre los dos personajes en materia educativa. De hecho, una lectura detallada la obra de cada uno de ellos muestra que las similitudes no son tantas ni tan próximas.

Existen motivos para pensar que las concepciones educativas de Rodríguez y Bolívar son bastante diferentes entre sí. En las próximas líneas se exploran estas diferencias con el objeto de mostrar que el pensamiento de Rodríguez no puede ser atado al de Bolívar como se ha hecho a lo largo de los últimos ciento cincuenta años. Para ello se divide la exposición en dos partes. En primer lugar se analizan las posibles similitudes en el pensamiento de ambos. A partir de este análisis se intentan determinar el alcance y significación de las mismas. Luego, en segundo lugar se da paso al estudio de aquello que se consideran diferencias en la forma de concebir la educación por parte de Bolívar y Rodríguez.

Las diferencias en la forma de concebir la educación son mucho más profundas de lo que se suele admitir. De esta manera tras el estudio de las similitudes y diferencias señaladas se procura fijar una postura en relación al vínculo pedagógico entre los autores que no se limite a meras suposiciones a partir de una serie de documentos. Para lograr nuestro objetivo realizamos un trabajo de análisis sobre las fuentes con las que contamos.

Es decir nos concentramos en el estudio de los textos conservados de Simón Rodríguez en los dos tomos que componen sus Obras completas (1999) y los discursos y proclamas de Bolívar compilados por la Biblioteca de Ayacucho (2007). En los escritos de ambos autores hay referencias a la relación entre política, república y la formación del pueblo. Según nuestro punto de vista del análisis de los textos es posible inferir que las posiciones de Rodríguez y Bolívar en relación a estos temas 
no sean tan similares, ni tan complementarias como la tradición acostumbra a sostener. De esta manera, basados en el estudio de las fuentes escritas de cada uno de los autores, pensamos que es posible poner en cuestión la interpretación tradicional que vincula el pensamiento de uno y otro como complementarios e indisociables.

\section{SUPUESTAS SIMILITUDES ENTRE RODRÍGUEZ Y BOLIVIAR EN MATERIA EDUCATIVA}

Las similitudes entre Rodríguez y Bolívar pueden circunscribirse de manera general a dos grandes temas: el rol del Estado en la educación del pueblo y la relación entre educación, política y economía. En relación al primer punto, los dos autores coinciden en la responsabilidad del Estado de hacerse cargo de la educación del pueblo. En diversos pasajes de la obra de Rodríguez y Bolívar puede observarse esta cuestión. En Extracto sucinto la obra Educación Republicana, Rodríguez (1999, TII, p. 227) escribe que la preocupación máxima del Estado es educar al pueblo para formar ciudadanos. A través de la educación el Estado debe transmitir los principios y valores fundamentales para vivir en una república (1999, TI, p. 229). Para ello es necesario un proyecto de educación popular, pensado, articulado y desarrollado por el propio Estado (1999, TI, p. 230). De acuerdo con su punto de vista, la responsabilidad del Estado en la educación del pueblo es tan grande que llega a identificar el triunfo de la revolución e independencia latinoamericana con la posibilidad de la educación de su pueblo.

Por su parte, Bolívar en los diversos textos en los que hace referencia a la cuestión educativa sostiene la responsabilidad exclusiva del Estado en la educación del pueblo. De acuerdo con su punto de vista, el Estado es el responsable por la educación del pueblo. A través de sus instituciones educativas forma a los futuros ciudadano electores y a los responsables de dirigir los destinos de la república.

$\mathrm{El}$ segundo punto de coincidencias se centra en la relación que ambos autores observan entre educación política y economía. Para los dos autores tanto el desarrollo económico de un estado, como su conformación política dependen en gran medida de la educación del pueblo. Un pueblo ilustrado es la condición necesaria de un pueblo próspero y republicano. En los textos de Rodríguez existen referencias relativas a esta cuestión. ${ }^{6}$ De hecho podría decirse sin temor a equivocarnos que la prosperidad económica y la vida republicana constituyen los pilares de su propuesta educativa de primeras letras (1999, TI, p. 370).

Bolívar también hace referencia a esta relación. En su escrito sobre educación establece una relación entre la constitución de un pueblo y la educación que este recibe.

Ahora bien, estas coincidencias no son exclusivas de Rodríguez y Bolívar. La responsabilidad del Estado en la educación del pueblo junto con la relación

6 Toda la obra de Rodríguez se articula en torno a estos conceptos. La mayoría de sus escritos hacen referencia a estos temas. En Las reflexiones (1999, TI, p. 200-202), en el Extracto de la obra Educación Republicana (1999, TI, p. 240-244), en Sociedades americanas (1999, TI, p. 335-350), en Desviación del rio Vincocaya (1999, TI, p. 467-470). 
entre economía y vida social, son temas compartidos por la tradición pedagógica de los siglos XVIII y XIX. Los escritos de los ilustrados españoles del siglo XVIII hacen referencia a estas cuestiones. La política ilustrada de los funcionarios españoles de la segunda mitad del siglo XVIII es clara en este punto. La relación entre responsabilidad estatal en la educación de los sectores populares, progreso y prosperidad social, se encuentra presente en la mayoría de los escritos producidos por ellos durante este período.

En función de ello se puede decir que estas coincidencias no pueden ser señaladas como una singularidad propia de Bolívar y Rodríguez. Es insuficiente apoyarse en estas similitudes para mostrar el estrecho vínculo teórico político que se pretende demostrar a lo largo de la historia, entre los dos autores en cuestión. No es correcto pensar en una simbiosis entre ellos por el simple hecho de compartir las mismas ideas que el resto de los intelectuales de la época. A su vez, por la misma experiencia de vida política e intelectual de Bolívar es arriesgado pensar que estas ideas sean responsabilidad exclusiva de Rodríguez o que el maestro tenga un rol fundamental en la adquisición de las mismas. La relación entre Rodríguez y Bolívar es muy esporádica a lo largo de sus vidas. Sus encuentros no superan en su conjunto un total de cinco años a lo sumo. Si bien la cantidad de años no determina la calidad del vínculo, la vida intelectual y política del Libertador puede entenderse y estudiarse independientemente de Rodríguez y viceversa. En el mejor de los casos podría decirse que ambos compartían los intereses de la época en materia educativa.

Siguiendo a Jesús Andrés Lasheras (2004), la calificación de Rodríguez como político y maestro ilustrado puede ser extendida, al menos en este punto, a la figura de Bolívar. A pesar de estas coincidencias, propias de la producción intelectual de la época, existen profundas diferencias entre Rodríguez y Bolívar en materia educativa. La más importante de ellas está relacionada en la forma con la que ambos autores se proponen llevar adelante la educación del pueblo.

La diferencia más importante entre Rodríguez y Bolívar en relación a la educación del pueblo reside en la metodología que cada uno propone para cumplir su objetivo. La mayoría de los autores al momento de abordar esta cuestión se refieren a la designación de Rodríguez como ministro de Educación de la república de Bolivia. ${ }^{7}$ Según ellos la designación por parte de Bolívar es una prueba incuestionable del apoyo de Bolívar al maestro y de su convicción y conocimiento acerca de la propuesta de Rodríguez. Castellanos (2007), hace referencia al envío por parte de Bolívar, del proyecto de educación popular de Rodríguez, para ser evaluado por las autoridades de Chuquisaca. A su vez, cita en el cuerpo del texto la respuesta de las autoridades de la ciudad en la que expresa la conformidad con el mismo. Ante este hecho resulta casi imposible dudar que Bolívar estuviese en desacuerdo o desconociera el proyecto de Rodríguez. Sin embargo, pensamos que existe una posibilidad de cuestionar esta afirmación.

7 El título exacto del cargo era director de enseñanza pública, de ciencias físicas, matemática y de artes; director general de minas, agricultura y caminos públicos (1999, TI, p. 84). 
En primer lugar el texto de la cita de Castellanos solo hace referencia al beneplácito de las autoridades de la presencia y responsabilidad asignada a Castellanos (2007, p. 149). La única referencia al proyecto es un tanto vaga y general. Nada nos lleva a pensar que el supuesto proyecto puesto al alcance de las autoridades solo haya sido una presentación general de las intenciones del maestro. De hecho no existe hasta el momento ningún documento que dé cuenta de cuál era el proyecto de Rodríguez. Sucre en una carta a Bolívar se queja amargamente que Rodríguez nunca le presenta un proyecto acabado. Según él, Rodríguez solo hace mención a vaguedades y generalidades ante el requerimiento de presentación del proyecto educativo (Rumazo González,1976, p. 149). ${ }^{8}$ La única referencia a la práctica educativa de Rodríguez en Chuquisaca se encuentra en la Defensa de Bolívar. Allí Rodríguez cuenta en una nota al pie de página en qué consiste su propuesta educativa y las razones de su fracaso.

Jesús Andrés Lasheras (2004) al analizar el fracaso del maestro en Bolivia lanza un lúcido interrogante. Según su interpretación, no queda tan claro que Bolívar estuviese al tanto de lo que el maestro va a llevar a cabo en el territorio boliviano con el nombre de educación popular. Para Lasheras el carácter radicalizado e intransigente de la propuesta de Rodríguez no es tenido en cuenta por Bolívar. Podría llegar a pensarse que ambos conocen aquello que los une es decir la necesidad de formar un pueblo republicano y el rol del Estado en esa construcción. Sin embargo, no es tan claro que Bolívar estuviese al tanto al detalle de la propuesta de Rodríguez. Es probable que el Libertador no aprobara la conducta e ideas que Rodríguez puso en práctica en Chuquisaca. Si hubiese estado al tanto de la totalidad del proyecto hay cuestiones que deberían explicarse con mayor precisión. Por ejemplo su actitud y falta de apoyo al accionar de Rodríguez en su cargo y su conducta tras el fracaso del maestro.

Bolívar y Rodríguez se separan en Bolivia tras unos meses de contacto y no se vuelven a ver. Cuando Rodríguez le escribe un año después para contar lo sucedido en Chuquisaca y explicar las causas de su salida de la república de Bolivia nunca recibe respuesta por parte de Bolívar. Tampoco lo convoca a ocupar ningún cargo de relevancia, ni lo consulta sobre ninguna materia. Tras su separación en Bolivia ninguno de los dos vuelve a tener contacto con el otro. Estos datos llevan a pensar que, al menos de parte de Bolívar, existe un distanciamiento de la figura de Rodríguez. Las causas de este distanciamiento son desconocidas, no obstante ello resulta sumamente significativo que haya ocurrido tras el fracaso de Rodríguez y su propuesta educativa. Es probable que este alejamiento marcara un posible desacuerdo por parte de Bolívar. No lo sabemos, pero lo dejamos planteado como una posibilidad no descabellada.

\section{DIFERENCIAS POLÍTICAS Y EDUCATIVAS RESPECTO DEL TIPO DE EDUCACIÓN DEL PUEBLO}

En función de lo que han escrito sobre educación es posible señalar algunas diferencias que no son de forma. Briceño Porras (1991) es uno de los pocos autores

8 Parte del texto de la carta es transcripto por Rumazo González (1976,p. 149). En dicho texto escribe: "Diferentes veces he pedido que Rodríguez me traiga por escrito el sistema que él quiere adoptar, para que me sirva de regla, y en ocho meses no lo ha podido presentar". 
que sostiene la existencia de estas diferencias. Según su punto de vista las propuestas de Rodríguez y Bolívar son diferentes aunque no contradictorias (Briceño Porras, 1991, p. 259). El autor señala una serie de diferencias entre las que destaca el apoyo de Bolívar a la pedagogía de Lancaster. A lo largo de los años Bolívar da muestras de su acercamiento a la propuesta lancasteriana, ya sea a través de la fundación de escuelas o el impulso económico y político que brinda al propio Lancaster y su escuela de Caracas. Bolívar como tantos otros políticos latinoamericanos ven en el "método de enseñanza mutua" la oportunidad de alfabetizar de manera sencilla y económica a grandes masas de la población. Desde sus años como conspirador en Londres traba contacto con la propuesta Lancasteriana durante 1810 en la casa de Miranda.

Briceño Porras (1991) y Weinberg (1995) entre otros sostienen que esta predilección de Bolívar por el método Lancaster solo se debe a las necesidades coyunturales de la América española. Según ellos, ante la necesidad de formar un pueblo ilustrado el método Lancaster se presenta como una oportunidad inmejorable para alfabetizar de forma masiva, en un tiempo relativamente corto y a un bajo costo a grandes masas de población. Más allá de las causas que motivan a Bolívar para adoptar desde los primeros años del siglo XIX el método Lancaster interesa indagar acerca de las implicancias políticas y filosóficas de ese método y la posición de Rodríguez ante las mismas. Ya que, allí pueden vislumbrarse una serie de diferencias significativas para el proyecto político que ambos autores pretenden llevar adelante.

Rodríguez se expresa de manera categórica contra la metodología Lancaster. Las razones que da para apoyar su postura son de carácter político y filosófico. Para el maestro, la metodología impulsada por Bolívar tiene una serie de consecuencias que atentan contra el proyecto político que se pretende establecer en América. En otras palabras, según Rodríguez, la metodología de la enseñanza mutua es contradictoria a la formación de un pueblo republicano en la medida que, lejos de formar ciudadanos autónomos, produce charlatanes en masa (Rodríguez, 1999, TII, p. 25). La crítica de Rodríguez se dirige a desnudar dos consecuencias directas de la aplicación del sistema en la formación de ciudadanos para las nuevas repúblicas. Ellas son la pasividad y docilidad de los educandos en su instrucción y la aplicabilidad de ideas ajenas a la realidad de los niños. Simón Rodríguez es muy contundente en este aspecto. Para él el método lancasteriano de "enseñanza muta es un disparate” (Rodríguez, 1999, TII, p. 25). Dicho "disparate” consiste, según el autor, en pretender que el proceso de enseñanza se centre en la repetición irreflexiva de los contenidos por parte de los niños.

Como señala en sus Consejos de amigo al Colegio de Lacatunga, Lancaster se propone repetir de memoria la Biblia y el efecto de dicha repetición es la formación de papagayos que no saben lo que dicen y se convierten en charlatanes (Rodríguez, 1999, TII, p. 25). La enseñanza memorística y repetitiva del sistema es inadmisible para Rodríguez. Desde sus primeros escritos, Rodríguez ataca la educación memorística. Según su punto de vista una enseñanza semejante solo es equiparable con la instrucción. La instrucción es insuficiente en la formación de un ciudadano. Ella es concebida como la transmisión de contenidos que son 
recibidos de manera irreflexiva por el alumno. En su obra distingue educar de instruir. Para Rodríguez la educación es un trabajo que exige crítica y comprensión de lo aprendido. A diferencia de Lancaster, la enseñanza excede ampliamente al trabajo memorístico y repetitivo de los alumnos. La comprensión y reflexión de lo enseñado es un elemento central en su propuesta. Por ello sostiene que enseñar "es hacer comprender. Es emplear el entendimiento, no hacer trabajar la memoria" (Rodríguez, 1999, TI, p. 399).

En Sociedades americanas se propone enseñar a ser preguntones a los niños para que no obedezcan ciegamente a la autoridad. Para Rodríguez, la capacidad crítica es indispensable para juzgar adecuadamente. Y esta, a su vez es imprescindible para la formación de una sociedad republicana. En este sentido escribe "todo lo bueno que hay en sociedad se debe a la crítica o mejor dicho, la sociedad existe por... Criterio es lo mismo que discernimiento, criticar es juzgar con rectitud" (Rodríguez, 1999, TII, p. 140). La formación de la capacidad de juzgar es uno de los objetivos más importantes en la propuesta de Rodríguez para la formación de un ciudadano. En tanto ciudadanos tienen el derecho y el deber a elegir a sus representantes a través de los diversos actos electorales que puedan tener lugar. De manera tal que la educación de los niños debe prepararlos, entre otras cosas, para realizar esta tarea de la mejor forma posible.

Con la propuesta de Lancaster pasa algo muy diferente. El proceso pedagógico se encuentra estructurado en la repetición memorística de los contenidos. Los niños aprenden el alfabeto dibujando en la arena letras que ven en una tarjeta impresa que sostiene el monitor designado. Una vez que memorizan esta escritura pasan a las hileras de pizarras donde reproducen el proceso, tantos días como necesitase para producir su avance. Luego se pasa a la escritura en tarjetas y finalmente en cuadernos. También se enseña numeración y las cuatro reglas aritméticas, siguiendo el mismo método de copia, reproducción y repetición de caracteres, números y operaciones (Bowen, 2001, p. 376-377).

La memoria y la repetición, fundamentos pedagógicos del sistema, se encuentran íntimamente ligados a una serie de técnicas disciplinarias que apuntan a la formación de un obrero dócil y productivo. Narodowski (2007, p. 137) sostiene que el método Lancaster produce una serie de transformaciones relativas al control y disciplina de los niños en relación al mercado capitalista de trabajo. Para el autor el método memorístico en los contenidos se articula a través de una serie de acciones que estimulan la competencia, vigilancia, delación, temor al castigo y ascenso en la escala jerárquica dentro del alumnado.

A diferencia de las posiciones ortodoxas de la iglesia anglicana, Lancaster se opone al castigo físico de los niños. En su lugar propone una serie de refuerzos positivos, que estimulan a los alumnos a mejorarse y auto-superarse en su proceso de aprendizaje. Estos refuerzos positivos se materializan en un conjunto de premios que van desde el lugar en la fila de bancos (los primeros lugares están ocupados por los que menos errores cometen), distintivos de cuero, diversos obsequios, puestos dentro de la jerarquía escolar y en algunos casos hasta dinero en efectivo. El máximo objetivo a alcanzar por un alumno es la designación como monitor, la máxima autoridad entre los niños, debajo del maestro. 
Los monitores son alumnos destacados que reciben instrucciones directas del maestro y se encargan de tomar la lección, control del trabajo y vigilar el comportamiento del alumnado (Narodowski, 2007, p. 139). Dentro de esta estructura el lugar alcanzado por un alumno en función de sus méritos se sostiene en la medida que el niño se mantuviese sin cometer errores significativos. Si su rendimiento escolar disminuye o su conducta empeora, pierde los beneficios obtenidos (Dussel y Carusso, 1999, p. 105). La promoción de una clase a otra, como los premios obtenidos son una resultante individual del trabajo particular de cada alumno. El sistema de premios, provisto por Lancaster, si bien elimina los castigos físicos, genera una serie de comportamientos centrados en el individualismo y la competencia entre los niños. Narodowski (2007, p. 137) sostiene al respecto que el método de enseñanza mutua fomenta, a través de los premios "la ambición, el deseo y la competencia en función de la posesión individual de logros". De acuerdo con el autor estas conductas son funcionales a las nuevas ideas que ven la luz tras la Revolución Industrial. Bowen (2001, p. 381), sostiene que el complejo sistema de premios y castigos está de acuerdo con el ideal burgués de recompensa, que se impone dentro de la sociedad industrial en evolución (Bowen, 2001).

Para Narodowski, la enseñanza de principios morales del utilitarismo no es contradictoria con medidas que estimulan la competencia, el individualismo y la ambición. Según su punto de vista, se trata de producir saberes y conductas inherentes a una nueva moral que gana poder entre las sociedades industriales europeas de fines del siglo XVIII. La pedagogía de Lancaster se ajusta a "la moral de los agentes de mercado, la moral inmanente a personas iguales que intercambian equivalentes sin otra finalidad que la del lucro, la que se sustenta gracias a la competencia y la ambición" (Narodowski, 2007, p. 138).

En líneas generales la mayoría de los trabajos dedicados al sistema lancasteriano suelen coincidir en la relación existente entre el proyecto económico burgués y la propuesta de la educación mutua. Según estos autores la metodología lancasteriana se encuentra íntimamente asociada al proceso de Revolución Industrial europeo. Los nuevos avances técnico-productivos exigen la formación de un personal capacitado y adaptado a las nuevas circunstancias (Weinberg, 1995, p. 203).La enseñanza mutua se presenta como un medio capaz de cumplir con estos requerimientos. Los niños escolarizados en estas escuelas, son formados desde muy temprano en un ambiente y metodología muy similares a las fábricas modernas. Grandes salones, estructura piramidal, el rol del monitor equiparado al de capataz, el premio del mérito y la introducción, en algunos casos, de la retribución monetaria, son algunas de las prácticas que equiparan a la escuela lancasteriana con las fábricas.

El alumno lancasteriano, no guarda grandes diferencias con el obrero disciplinado premiado en función de sus propios logros en base al esfuerzo personal. La articulación de la escuela con el mundo del trabajo es una preocupación fundamental del método Lancaster. Nadorowski (2007, p. 146) sostiene que uno de los principales problemas a resolver por el método es la cuestión del disciplinamiento del trabajador y la participación futura de los niños en la actividad productiva. El sistema lancasteriano sostiene que la escuela debe preparar a los niños en la práctica de hábitos morales para formar hombres virtuosos y miembros útiles de la sociedad. Esta virtud y utilidad se materializa en su práctica como obreros fabriles dóciles y 
disciplinados. Podría decirse que la enseñanza mutua, prepara y disciplina al futuro obrero de la sociedad industrializada del capitalismo de fines del siglo XVIII y XIX.

En función de lo escrito puede observarse al menos tres diferencias sumamente significativas entre la propuesta de Rodríguez y la de Lancaster. Estas se vinculan con el objetivo perseguido por cada uno de los autores en la educación del pueblo, los valores promovidos y las consecuencias político intelectuales de la adopción de una y otra propuesta. La primera diferencia se encuentra asociada a la distancia existente entre el objetivo perseguido por Rodríguez y Lancaster con sus propuestas educativas. Rodríguez, lo escribe a lo largo de toda su obra reiteradas veces. La educación de primeras letras en América se propone la formación de un pueblo republicano. Mientras que Rodríguez asume y afirma a sus alumnos como ciudadanos activos de una república, Lancaster no. Rodríguez educa a sus alumnos como ciudadanos y los prepara con el fin de que puedan vivir en una sociedad republicana. Rodríguez supone a sus estudiantes como hombres participativos, creativos, reflexivos, dueños de su destino y en ejercicio pleno de sus derechos y obligaciones en tanto ciudadanos de una república

Lancaster, por su parte, se limita a la formación de un obrero productivo y fundamentalmente disciplinado. A lo largo de su propuesta es posible ver cómo concibe a su estudiante solo como un eslabón constitutivo de la cadena de producción industrial. El eslabón más simple y bajo de la cadena, el obrero. En sus escritos no hay referencia alguna a la participación de sus estudiantes en la política de su país, independientemente del lugar asignado en la producción. La virtud y utilidad destacada por Lancaster en sus escritos se relaciona con el adecuado y sumiso desempeño del lugar que les toca en la sociedad. A diferencia de Rodríguez, cuyos escritos poseen numerosas referencias a la participación política del pueblo, los textos de Lancaster se limitan a referencias morales relativas a la religión, la caridad y el mercado. Su propuesta es funcional a los sectores económicos y políticos de la burguesía industrial que gana terreno desde fines del siglo XVIII. Independientemente de los cambios implícitos en su proyecto educativo y de los supuestos fines filantrópicos del mismo, el lugar subalterno de los sectores populares no se pone en cuestión.

Por otro lado, los valores que promueven son contradictorios con la propuesta del maestro venezolano. Solo basta leer la obra de Rodríguez para darse cuenta que, valores tal como individualismo, ambición y competencia desmedida, son contraproducentes dentro de su proyecto político, económico y educativo. Difícilmente Rodríguez estuviese de acuerdo con una educación que promueva semejantes valores. Según su punto de vista dichos valores son la causa fundamental del fracaso del sistema republicano. En el Extracto sucinto sobre mi obra de educación republicana escribe sobre el este tema del individualismo. Allí sostiene que "al sublime precepto de 'ver en los intereses del Prójimo los Suyos propio', se sustituye la máxima más perversa que pueda haber inventado el egoísmo 'Cada uno para Sí y Dios para Todos" (Rodríguez, TI, 1999, p. 229). De acuerdo con su interpretación, esta actitud atenta contra uno de los principios fundamentales del sistema republicano tal cual como él lo entiende, a saber los deberes sociales. Así escribe que si el objetivo es constituir una república entonces "debemos emplear medios tan nuevos como es nueva la idea de ver por el bien de todos” (Rodríguez, TI, 1999, p. 229). 
La cooperación colectiva en lugar de los intereses particulares, puede observarse también en sus escritos en los que menciona ciertos aspectos económicos a ser tenidos en cuenta. Sobre la producción sostiene que "por máxima reguladora de la economía dígase antes de emprender: los productores se han de consultar para no producir más de lo necesario" (Rodríguez, TI, 1999, p. 240). La competencia desmedida solo trae desgracias y fracasos al sistema político republicano. Para consolidarlo hace falta en primer lugar un proyecto de educación popular asentado sobre principios políticos adecuados y valores tales como la cooperación, el trabajo colectivo y el conocimiento de los deberes sociales.

Como puede observarse la propuesta de Rodríguez guarda diferencias sustanciales con la de Lancaster. En este sentido, pensamos que es muy poco probable que ambas propuestas puedan pensarse de forma complementaria. Es más, sin mucho esfuerzo es posible sostener que son en gran parte contradictorias entre sí. Las ideas de Bolívar y las de Rodríguez se contraponen sustancialmente, en la medida que uno apoya explícita y materialmente al método de Lancaster con todo lo que ello implica y el otro no. En este sentido, no es posible sostener, como gran parte de la tradición hace, que nuestros autores comparten las mismas ideas educativas. Las únicas similitudes existentes entre ambos son las que ya hemos señalado. Estas similitudes lejos de ser concebidas como particularidades fuertes del pensamiento de Bolívar y Rodríguez, son aspectos ampliamente difundidos por todos aquellos hombres que piensan la educación entre los siglo XVIII y XIX.

\section{CONCLUSIÓN}

En función de lo escrito pueden desprenderse dos cuestiones importantes. La primera de ellas es que el pensamiento de Rodríguez debe ser considerado separadamente del de Bolívar. La obra de Rodríguez tiene un peso específico propio que merece ser estudiado y analizado en su particularidad. La segunda de ellas es que las ideas de Rodríguez son distintas a las de Bolívar, sobre los temas aquí analizados. No obstante, a pesar de esto, no es adecuado negar la relación y participación política de ambos dentro del proyecto republicano a gestarse en América. Ambos autores son republicanos y piensan que la educación del pueblo juega un papel fundamental en el triunfo y consolidación de dicho sistema. En este sentido puede decirse que Bolívar y Rodríguez pertenecen a un mismo grupo político. Ahora, sus diferencias aparecen cuando cada uno escribe qué entiende por educación y república.

Allí los escritos de los autores se distancian de manera notoria y contrapuesta. Al punto tal que nos resulta imposible pensar de la misma manera que Briceño Porras, quien afirma que las ideas de Bolívar y Rodríguez en materia educativa son diferentes pero no contradictorias. Es más, en muchas ocasiones ellas mismas son contradictorias entre sí. Las consideraciones acerca del pueblo, la ciudadanía, la división de cámaras, como así también sus proyectos educativos guardan profundas diferencias.

Podrá decirse, ensayando una última defensa a esta interpretación tradicional, que el gesto del Libertador de nombrarlo como ministro de Educación de Bolivia, es una prueba suficiente para demostrar el estrecho vínculo teórico y práctico entre 
ellos. A pesar de la veracidad de este hecho, pensamos que la lectura cuidadosa del mismo puede darnos algunas pistas para suponer lo contrario. En primer lugar, es cierto que Bolívar nombra a Rodríguez como funcionario de la república. Sin embargo cabe preguntarnos si el Libertador está al tanto de la radicalidad de los planes de Rodríguez. Es decir, cabe preguntarse si Bolívar conoce al detalle lo que Rodríguez se propone llevar a delante. Autores como Castellanos (2007), Briceño Porras (1991), sostienen que en su viaje al Alto Perú, los dos personajes mantienen profundas charlas acerca del mismo. Castellanos, en particular sostiene que los decretos educativos que Bolívar escribe durante este período son inspiración del propio Rodríguez.

Esto parece poco probable. En primer lugar, no hay prueba alguna que haga suponer la veracidad de esta afirmación. Castellanos escribe en condicional y solo hace referencia a los mismos como un posible hecho. En segundo lugar, en los escritos pedagógicos de Bolívar y decretos, puede verse que muchos de ellos hacen referencia a la creación de escuelas lancasterianas. ${ }^{9}$ Dada la apreciación de Rodríguez sobre este sistema, es poco probable que el maestro haya inspirado esos decretos.

Otro dato interesante es la supuesta falta de un proyecto educativo por parte de Rodríguez. Sucre se refiere al mismo en una carta a Bolívar. En ella escribe que a pesar de haberle pedido reiteradas veces la presencia del mismo, Rodríguez no cumple con el pedido. De hecho al momento de producirse su dimisión y posterior partida de Bolivia no se produce dicha presentación. Las cartas entre Sucre, Bolívar y Rodríguez son el único documento que se tiene para reconstruir este hecho. De la lectura de las mismas, parecería desprenderse que ninguno de los funcionarios está al tanto de ese proyecto de educación. Es más, Sucre pone como un hecho relevante de su conducta para con el maestro su relación con el Libertador.

Producto de este intercambio de posiciones Rodríguez presenta su renuncia al cargo. En una carta a Bolívar escrita el 30 de septiembre de 1827 en Oruro, el maestro da cuenta de su renuncia y del mal trato recibido por Sucre (Rodríguez, 1999, p. 131-136). En ella hay un dato que nos parece relevante a los fines que nos ocupan. Allí escribe, que él no debía darle explicaciones a nadie. En este sentido afirma: "yo no era un empleadillo adocenado de los que obstruyen las antecámaras: yo era el brazo derecho del Gobierno: yo era el hombre que U. había honrado y recomendado en público repetidas veces: yo estaba encargado de dar ideas no de recibirlas" (Rodríguez, 1999, p. 133).

Por último, queda por analizar la supuesta reacción de Bolívar tras la renuncia de Rodríguez a su cargo. Sobre la misma se señalan tres puntos. En primer lugar, Bolívar nunca responde las cartas que Rodríguez le envía, no solo para contar su punto de vista respecto de la disputa con Sucre, sino tampoco aquellas en las que pide ayuda. Tampoco vuelven a verse. No hay dato alguno que permita sospechar que Bolívar haga algún tipo de intento de acercamiento con su supuesto antiguo

9 Salcedo Bastardo recoge varios decretos dedicados a la educación en El primer deber (1973). Entre ellos es digno de mención el decreto del 31 de enero de 1825 en la ciudad de Lima. El mismo se titula: "En cada capital de departamento una escuela normal lancasteriana" y entre los considerandos figuran la eficacia y celeridad del sistema lancasteriano y la necesidad de extenderlo a todo el territorio (Salcedo Bastardo, 1973, p. 321). 
maestro.Y, por último, no se observan entre los documentos ninguna defensa pública hacia Rodríguez por parte de Bolívar. En definitiva pareciera ser que la posición del Libertador está más próxima a la de Sucre que a la de Rodríguez.

En relación a los documentos y pruebas existentes quedan interrogantes, más que certezas. ¿Cuáles son los motivos o las causas de esta reacción? ¿Es probable que Bolívar considerara excesivas las prácticas de Rodríguez en materia educativa? ¿Considera demasiado radicalizada la conducta de Rodríguez?

Tal vez nunca se tenga respuesta a los mismos. Sin embargo su reacción y conducta hacia Rodríguez podría haber sido muy distinta a la que tuvo, si sus ideas hubiesen sido tan próximas. Si hubiese existido tal comunión de ideas, como la que se intenta señalar, pensamos que algún tipo de defensa o atención podrían haberse ensayado. Sin embargo, solo tenemos el silencio y la indiferencia política del Libertador hacia Rodríguez, a partir de 1826.

De acuerdo con lo escrito, no hay elementos que permitan continuar con la posición tradicional relativa a la comunión de ideas entre Rodríguez y Bolívar. En función de lo escrito, se sostiene que el pensamiento de madurez de Rodríguez se diferencia sustancialmente de Bolívar. Existen marcadas diferencias entre sus formas de pensar el sistema republicano, el rol de los ciudadanos en dicho sistema, como así también en la composición y educación del pueblo. No se trata de diferencias mínimas que pueden ser zanjadas con una simple lectura forzada de los textos. Las diferencias respecto del sistema republicano y la educación del pueblo son profundas. A partir de las mismas se desprenden dos formas distintas de considerar al pueblo y su participación política. Bolívar piensa un pueblo heterogéneo compuesto por el populacho y por el pueblo virtuoso e ilustrado.

En función de esta división plantea una división política entre ciudadanos activos y pasivos. Semejante decisión establece una línea divisoria entre aquellos que solo pueden elegir quién los iba a mandar y aquellos que pueden ser electos para desempeñar funciones dentro del Estado (Bolívar, 2007, p. 92). Rodríguez (1999, TI, p. 271, p. 324, p. 382; TII, p. 275, p. 289), por su parte, considera un pueblo homogéneo, constituido por todos los ciudadanos de la república. Nunca hace referencia a una división que establezca algún límite en la participación política de los mismos. A diferencia de Bolívar, la posición de Rodríguez no establece división alguna entre los que desempeñan funciones políticas representativas y los representados. En otras palabras, no hay demarcación entre los que mandan y obedecen. Mejor dicho, no fija ningún límite para que el pueblo en su totalidad pueda desempeñar funciones representativas en todos los niveles. De hecho, a lo largo de sus escritos, puede observarse con claridad esta postura. Su posición respecto de las cámaras del parlamento es un dato contundente que confirma esta posición. Su oposición a la división de Cámaras, juntos con los argumentos que brinda, muestra explícitamente su concepción del pueblo y la república.

Por otro lado, la posición de ambos pensadores en relación a la educación del pueblo presenta diferencias considerables e irreductibles. Las posturas de Bolívar y Rodríguez conciben al educando y su formación de dos formas diferentes. Esto puede verse explícitamente a través de la lectura de la obra de Rodríguez. No solo a través de las críticas al sistema lancasteriano que impulsa y apoya Bolívar, sino también a partir de su propuesta positiva. 
Mientras un sistema propone un alumno competitivo, pasivo y adaptado a la repetición memorística de los saberes, el otro se propone la formación de un alumno solidario, activo y reflexivo. Como sostiene Ortega (2011), la propuesta de Rodríguez y la de Lancaster difieren significativamente en relación al tipo de ciudadano que aspiran a formar. Por este motivo sugiere el autor, la propuesta de Rodríguez encuentra tantas dificultades para su aplicación y desarrollo, mientras que de la Lancaster no. A lo largo del capítulo se ha hecho referencia a que ambas propuestas suponen un alumnado particular y en función del mismo plantean sus objetivos y metodología de trabajo. Las mismas, son bien diferentes entre sí. Una simple lectura de los textos lancasterianos y de la obra de Rodríguez basta para dar cuenta de las marcadas y profundas diferencias que existen entre ellas.

De manera tal que si se tiene en cuenta el apoyo que Bolívar brinda al sistema lancasteriano, lo favorable al mismo en materia de política educativa, sus referencias elogiosas y sus escritos sobre educación, puede inferirse que su postura en relación a la educación del pueblo se diferencia de la de Rodríguez. El único punto de contacto que tal vez pueda ser rescatado es que ambos personajes están a favor de la educación del pueblo. Pero más allá de ello difieren acerca de la forma, contenido, metodología y sentido de esa educación. Las propuestas de Rodríguez y Bolívar, si tenemos en cuenta lo escrito a lo largo de este capítulo, distan mucho de ser idénticas, similares o tan siquiera complementarias. Es más pensamos que su lectura y análisis arrojan más punto de divergencias que ideas comunes. En este sentido pensamos que es posible poner en cuestión la lectura tradicional acerca de la relación de Bolívar y Rodríguez. En función del estudio realizado pensamos que hay elementos más que suficientes para decir que la relación entre Bolívar y Rodríguez está signada por más desencuentros que encuentros.

En función de lo escrito se sostiene que es poco rigurosa la idea que sostiene que Bolívar es formado desde su infancia, por un maestro revolucionario y republicano, para convertirse en el Libertador de América. De la misma manera debe ponerse en cuestión la idea de una suerte de comunión teórico política entre ambos, tras las guerras de independencia. Más allá del afecto y reconocimiento que ambos personajes se manifiestan a lo largo de sus vidas, sus ideas, pensamientos y propuestas políticas deben ser considerados independientemente una de la otra.

\section{REFERENCIAS}

Achury Valenzuela, D. Azar e infortunio de Don Simón Rodríguez. Bogotá: [s.n.],1954. Álvarez Freites, M. Simón Rodríguez tal cual fue. Vigencia perenne de su magisterio. Caracas: Ediciones del Cuatricentenario de Caracas, 1966.

Amunátegui, M. L. Ensayos biográficos. Santiago de Chile: Imprenta Nacional, 1876. André, M. Bolivar y la democracia. Barcelona: Editorial Araluce, 1924.

Bolívar, S. Proclamas y discursos. Caracas: Ediciones de la Biblioteca de Ayacucho, 2007. Bowen, J. Historia de la educación occidental. Tomo III: El occidente moderno. Europa y el Nuevo Mundo, siglos XVII-XX. Barcelona: Herder, 2001.

Briceño Porras, G. El extraordinario Simón Rodríguez. Caracas: Fondo Editorial IPASME, 1991. 
BRIGgs, R. Tropes of the enlightenment in the age of Bolivar: Simón Rodriguez and the American essay at revolution. Nashville: Vanderbilt University Press, 2010.

Castellanos, R. R. Simón Rodríguez, pensador universal y pulpero de Azángaro. Caracas: FOGADE, 2007.

Cova, J. A. Don Simón Rodríguez, maestro y filósofo revolucionario. Primer socialista americano. Buenos Aires: Editorial Venezuela, 1947.

Dussel, I.; Caruso, M. La invención del aula. Buenos Aires: Santillana, 1999.

Grases, P. Los escritos de Simón Rodríguez. Caracas: [s.n.], 1953.

Jáuregui OlazÁbal, M. Vida y obra de Don Simón Rodríguez. Mérida: ULA/CEP, 2000.

Lasheras, J. A. Simón Rodríguez: maestro ilustrado y político socialista. Caracas: Universidad Nacional Experimental Simón Rodríguez, 2004.

Lee Lofstrom, W. La presidencia de Sucre en Bolivia. Caracas: Academia Nacional de la Historia, 1987.

Lozano y Lozano, F. El maestro del Libertador. París: Ollendorff, 1913.

Mancini, J. Bolivar y la emancipación de las colonias españolas desde los orígenes hasta 1815. París: C. Bouret, 1914.

Molins, M. La República y la educación en Simón Bolivar y Simón Rodríguez y su proyección actual. Caracas: Universidad Central de Venezuela; EBUC, 1998.

Narodowski, M. Infancia y poder. Buenos Aires: Aique, 2007.

O'Leary, D. F. Memorias del general O'Leary, publicadas por su hijo, Simon B. O'Leary, por órden del gobierno de Venezuela y bajo los auspicios de su presidente, general Guzman Blanco. Caracas: Imprenta de la Gaceta Oficial, 1879-1888.

Ortega, F. Tomen lo bueno dejen lo malo: Simón Rodríguez la educación popular. Revista de Estudios Sociales, Bogotá: Universidad de los Andes, n. 38, p. 30-46, 2011.

Rodríguez, S. Obras completas. Caracas: Presidencia de la República, 1999. v. I y II.

Rojas, A. Simbiosis de los dos Simones. Socialismo desde el ALBA. Maracay: [s.n.], 2008.

Rumazo González, A. Ideario de Simón Rodríguez. Caracas: Ediciones Centauro, 1976.

Salcedo Bastardo, J. L. El primer deber. Caracas: Ediciones Equinoccio, 1973.

Vera Peñaranda, P. Simón Rodríguez y sus ideas de educación popular en Bolivia. La Paz: IEB, 2009.

Weinberg, G. Modelos educativos en la historia de América Latina. Buenos Aires:AZ, 1995.

\section{SOBRE EL AUTOR}

Maximiliano Durán es doctor en filosofía por la Universidad de Buenos Aires (Argentina). Professor de la misma instituición.

E-mail: maximiliano1771@gmail.com 